\title{
Cold torus whistlers: An indirect probe of the inner Jovian plasmasphere
}

\author{
K. Wang ${ }^{1}$ and R. M. Thorne \\ Department of Atmospheric Sciences, University of California, Los Angeles \\ R. B. Horne \\ British Antarctic Survey, Natural Environment Research Council, Cambridge, England \\ W. S. Kurth \\ Department of Physics and Astronomy, University of Iowa, Iowa City
}

\begin{abstract}
The dominant features of Jovian whistlers observed by Voyager 1 in the inner cold torus around $R_{J} \approx 5.3$ are studied using the technique of ray tracing with an offset tilted dipole. These whistlers are unducted, and the electron density in the inner plasmasphere plays a controlling role in the access of whistlers to the cold torus. The upper frequency cutoff of inner zone whistlers allows a firm limit to be placed on the minimum electron density $3-5 \mathrm{~cm}^{-3}$ at higher latitudes in the inner plasmasphere around $L \approx 5.3$, a region where no spacecraft have ever been. Backward ray tracing from the observation location is used to determine the source location at the top of the Jovian atmosphere, and it is shown to be consistent with the latitude of lightning events observed by Voyager 1 .
\end{abstract}

\section{Introduction}

Lightning-generated Jovian whistlers have been observed [e.g., Gurnett et al., 1979; Gurnett and Scarf, 1983] in three distinct locations, which are referred to here and in previous papers as regions A, B, and C. The regions of detection along the Voyager 1 trajectory are shown in Figure 1. The majority of Jovian whistlers were confined to region $B$ in the inner cold torus $5.25 \leq L \leq 5.6$ [Kurth et al., 1985]. These whistlers are characterized by much shorter dispersion (ranging from 40 to $70 \mathrm{~s} \mathrm{~Hz}^{1 / 2}$ ) compared with those observed in regions $A$ and $C$ in the warm torus. Consequently, the waves which reach region $B$ must have propagated through regions where the path-integrated electron densities were much lower. Plasma in the inner torus is too cold to accurately analyze the dispersion of the majority of observed whistlers. Although information on the equatorial proton concentration and the ion parallel temperature (scale height) can be extracted from a dispersion analysis of whislters in the warm torus [e.g., Crary et al., 1996; Wang et al., this issue], the dispersion observed in region $B$ is insensitive to changes in these key plasma parameters. Nonethe-

\footnotetext{
${ }^{1}$ Now at Jet Propulsion Laboratory, Pasadena, California.

Copyright 1998 by the American Geophysical Union.

Paper number 98JA00965.

0148-0227/98/98JA-00965 $\$ 09.00$
}

less, important features of whistlers observed in the cold torus have not been adequately explained in previous studies. Specifically, the present study will address the question of why most whistlers were confined to this inner region and why the observed location for detection spanned a range from $L=5.25$ to $L=5.6$, instead of being centered around the density peak at $L=5.3$, as would be expected if the density peak guides whistlers from the ionosphere to the observed equatorial region [Wang et al., 1995]. To simulate the observed characteristics of region $B$ whistlers, a new electron density model has been constructed for the inner Jovian magnetosphere. The density model is used in the HOTRAY code [Horne, 1989] to trace waves back to their source location in the Jovian ionosphere on the assumption that the waves are unducted. This source location is consistent with the latitude range over which optical lightning flashes were observed by Voyager 1 . Our analysis also yields information on structure of the inner torus and places a strict limit on the electron density at intermediate latitude in the inner plasmasphere, a region that currently is poorly defined due to lack of in situ satellite measurements.

\section{Observed Features of Whistlers in the Inner Cold Torus}

Figure 2 shows a frequency-time spectrogram of observed whistlers in the cold torus; the properties of such whistlers have been extensively described by Kurth et al. [1985]. Wave dispersion is very short for each of the 


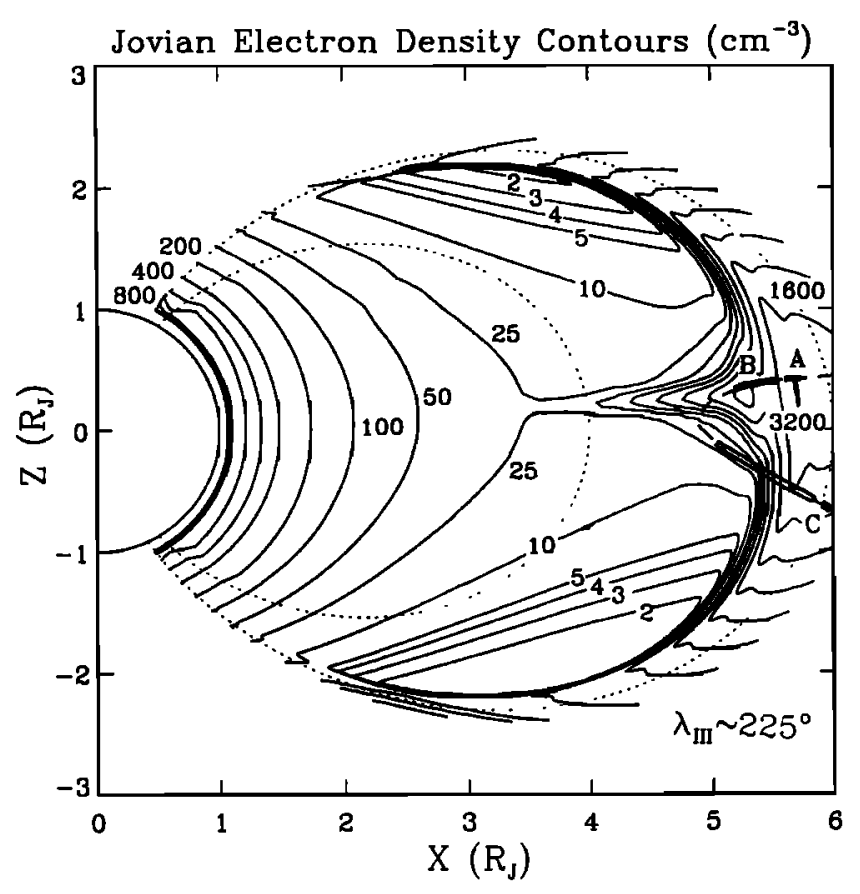

Figure 1. Optimum electron density contours (solid lines) in the inner plasmasphere required to account for the features of region $B$ whistlers. The Voyager 1 trajectory through the Io torus is indicated by a bold dashed line, on which the regions $A\left(\lambda_{\text {III }} \approx 200^{\circ}\right)$, $\mathrm{B}\left(\lambda_{\text {III }} \approx 225^{\circ}\right)$, and $\mathrm{C}\left(\lambda_{\text {III }} \approx 330^{\circ}\right)$ of whistler detection are also shown.

whistler events (40-70 $\left.\mathrm{s} \mathrm{Hz}^{1 / 2}\right)$. The frequency range of whistlers observed in region B is summarized in Figure 3 as a function of $L$ shell and SCET (spacecraft event time). While the data exhibit considerable variability, the lowest upper cutoff frequency $(3 \mathrm{kHz})$ was observed near SCET 0944 when the spacecraft was inbound near $L=5.5$, and the highest upper cutoff frequency $(8 \mathrm{kHz})$ was observed around $L=5.3$ near SCET 1016. During this time interval, the observed upper cutoff frequencies exhibited a tendency to increase as, the arrow indicates. This trend for the upper cutoff frequency to increase with decreasing $L$ is not due to any deficiencies of the instrument. The dynamic range of broadband electric field strength does not change during this time period [Kurth et al., 1985]. Wideband data were only available for certain time periods, indicated by the solid bars at the top of the figure. Discrete whistlers were observed during most of these intervals of wideband coverage. Whistler occurrence is probably high throughout the inner torus.

\section{Models}

The access of Jovian whistlers to region $B$ and the observed spectral properties of the waves have been studied using the HOTRAY code [Horne, 1989] with a realistic electron density model for the inner Jovian magnetosphere. This composite model contains components to represent the ionosphere, the inner plasma- sphere, and the Io torus as described in a companion paper [Wang et al., this issue]. Certain key parameters in each model were varied to obtain the optimum density model consistent with whistler observations. Both the access of waves to locations of observation and wave dispersion (where appropriate) were used to define this optimum electron density model. The offset and tilt of the dipole (OTD) are taken into account to explore the source latitudinal locations in terms of Jovigraphic coordinates. The composite contours of electron density of our optimum model, corresponding to the System III longitude $\lambda_{\text {III }}=225^{\circ}$ where region $B$ whistlers were observed in the cold torus, is shown in Figure 1. There is a pronounced minima in electron density at middle latitudes in the inner plasmasphere (specifically note the contours of electron density at $2,3,4,5$, and $10 \mathrm{~cm}^{-3}$ ). The local plasma frequency in this region plays a controlling role in determining the upper frequency cutoff [Gurnett et al., 1981; Wang et al., 1995] for whistlers which have access to region $B$. There was a prolonged period along the outbound Voyager trajectory where no wideband data were available south of the magnetic equator, as indicated by the open box in Figure 1. This absence of wideband data spans the entire $L$ range where region B whistlers were detected on the inbound orbit.

\section{Ray Paths of Whistlers}

The composite density model was incorporated into the HOTRAY code, and waves were traced backward from the observation point in region $B$ to the ionosphere. Only waves with wave normal angles approximately vertical to the planetary surface at the base of the ionosphere were considered in this study, since this is the most probable condition for whistlers to escape from the ionosphere [e.g., Helliwell, 1965]. Figure 4 illustrates how the appropriate ray path was chosen for the case of $3-\mathrm{kHz}$ waves observed by Voyager near $L=5.3$. Waves were assumed to be unducted and to propagate at a wave normal angle $\psi$ to the ambient magnetic field. Six ray paths are shown for waves with a propagation angle $\psi_{i}$ at the observation location at the inner portion of region $\mathrm{B}(L=5.3)$ and corresponding wave normal angle $\psi_{f}$ at the ionosphere. The ionospheric entry latitudes are represented by $\lambda_{m 0}$, and the difference between $\psi_{f}$ and $\psi_{f}^{*}$ (the wave angle which is vertical to the ionosphere) is tabulated as function of $\psi_{i}$ in the bottom portion of the figure. In this specific case the source location in the Jovian ionosphere was determined to be near $\lambda_{m}=53.5^{\circ}$, and the wave normal angle at the observation point was $\psi_{i} \approx 142^{\circ}$, corresponding to the minimum value of $\left|\psi_{f}-\psi_{f}^{*}\right|$.

Figure 5 shows sample ray paths within the observed frequency ranges $(2-8 \mathrm{kHz})$ for whistlers that are able to propagate into the cold torus over the range (5.3 $\leq L \leq 5.5)$. Typical source latitudes in the ionosphere range from $53.5^{\circ}(L \approx 2.8)$ to $58^{\circ}(L \approx 3.5)$. Waves propagate substantially across field lines from the source location near $L=3.0$ in the ionosphere to 


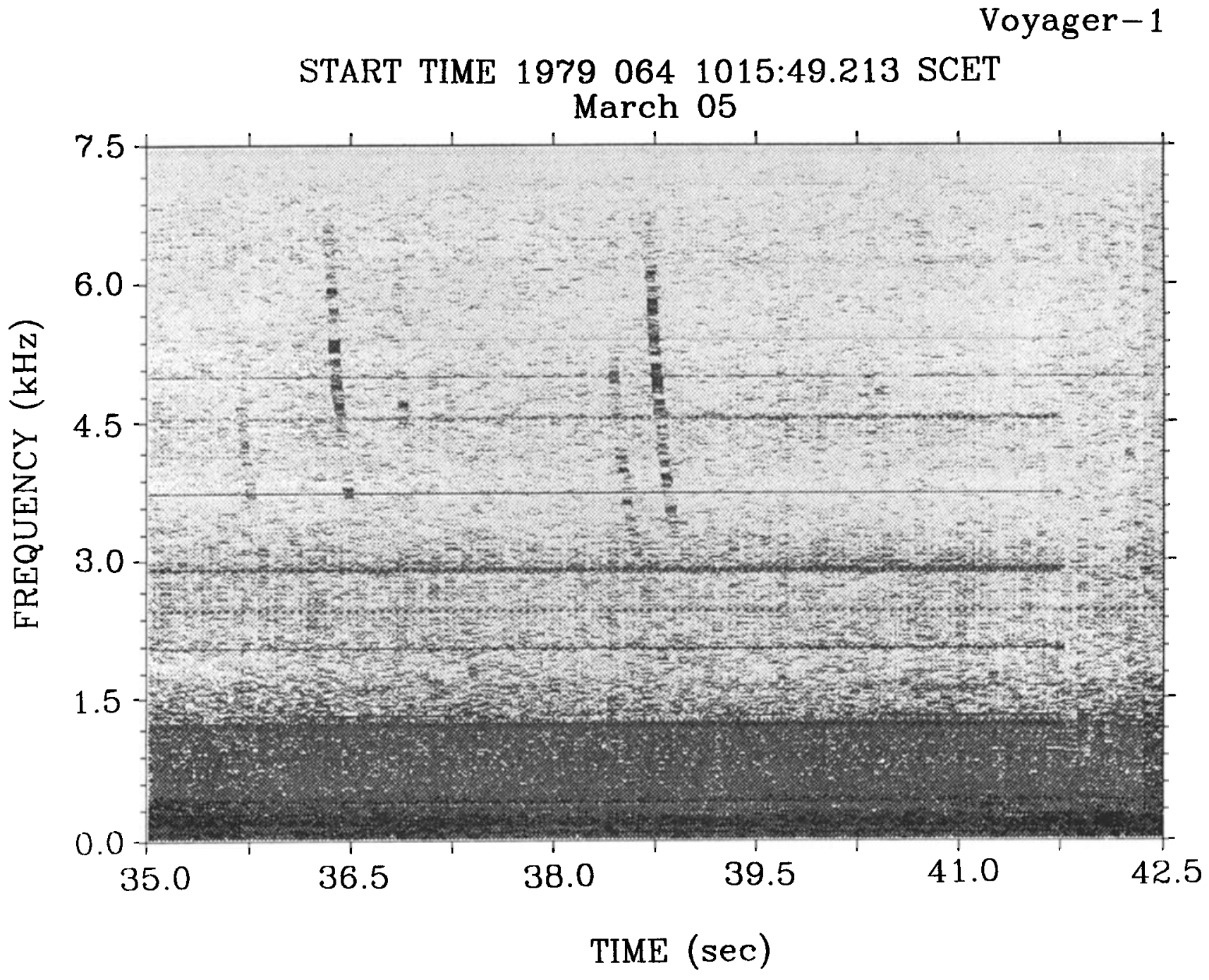

$$
\mathrm{R}=5.28 \mathrm{R}_{\mathrm{J}} \text { Lat }=-5.68^{\circ} \quad \lambda_{\mathrm{III}}=225.47^{\circ}
$$

Figure 2. An example of frequency-time spectrogram of whistlers observed in the cold torus. The dispersion is approximately $40 \mathrm{~s} \mathrm{~Hz}^{1 / 2}$ over the frequency range from 3 to $7 \mathrm{kHz}$.

$L=5.3$ at the observation point near the equatorial plane. The highest observed frequency waves $(8 \mathrm{kHz})$, which only have access to the inner portion of region $\mathrm{B}$, are refracted considerably at intermediate latitudes along the $L=5.3$ field line. In contrast, $3-\mathrm{kHz}$ waves observed in the outer portion of region $B$ are refracted to a much lesser extent. The stronger refraction of 8$\mathrm{kHz}$ waves is caused by the lower plasma density (and hence plasma frequency) at middle and high latitudes in the inner plasmasphere (Figure 1). This refraction is enhanced by the finite angle of propagation, which effectively excludes waves from locations where the wave frequency is above about one third of the plasma frequency. Waves with lower frequencies, such as $2-\mathrm{kHz}$ and $3-\mathrm{kHz}$ waves (dashed lines), can propagate through this lower density region with little refraction. However, these waves are refracted by the strong gradient between the cold torus and the warm torus near the equator and arrive at region $\mathrm{B}$ near $L=5.5$ (dashed lines). The inner plasmaspheric densities shown in Figure 1 were scaled up from the values given by Divine and Garrett [1983] to allow $8-\mathrm{kHz}$ waves to access the inner portion of region $B$ where they have been observed. Figure 1 presents the optimum density model to explain the observations of $8-\mathrm{kHz}$ waves.

The ionospheric source location for different frequency whistlers is explored in Figure 6, which shows ray paths plotted as a function of invariant Jovigraphic latitude $\left(\Lambda_{g}\right)$ versus magnetic latitude $\left(\lambda_{m}\right)$. The $L$-shell range for whistler detection on Voyager (near $\lambda_{m}=0^{\circ}$ ) in regions A and B is indicated by bold solid lines. Eightkilohertz $\mathrm{kHz}$ waves (top left panel) launched from $\Lambda_{g}=40.5^{\circ}, 41.5^{\circ}$ (dotted lines) cannot access region B; they propagate into the equatorial region at $L$-shells less than 5.3. Waves launched from $\Lambda_{g}=42^{\circ}$ to $43^{\circ}$ (solid lines) are able to access the observation point in 


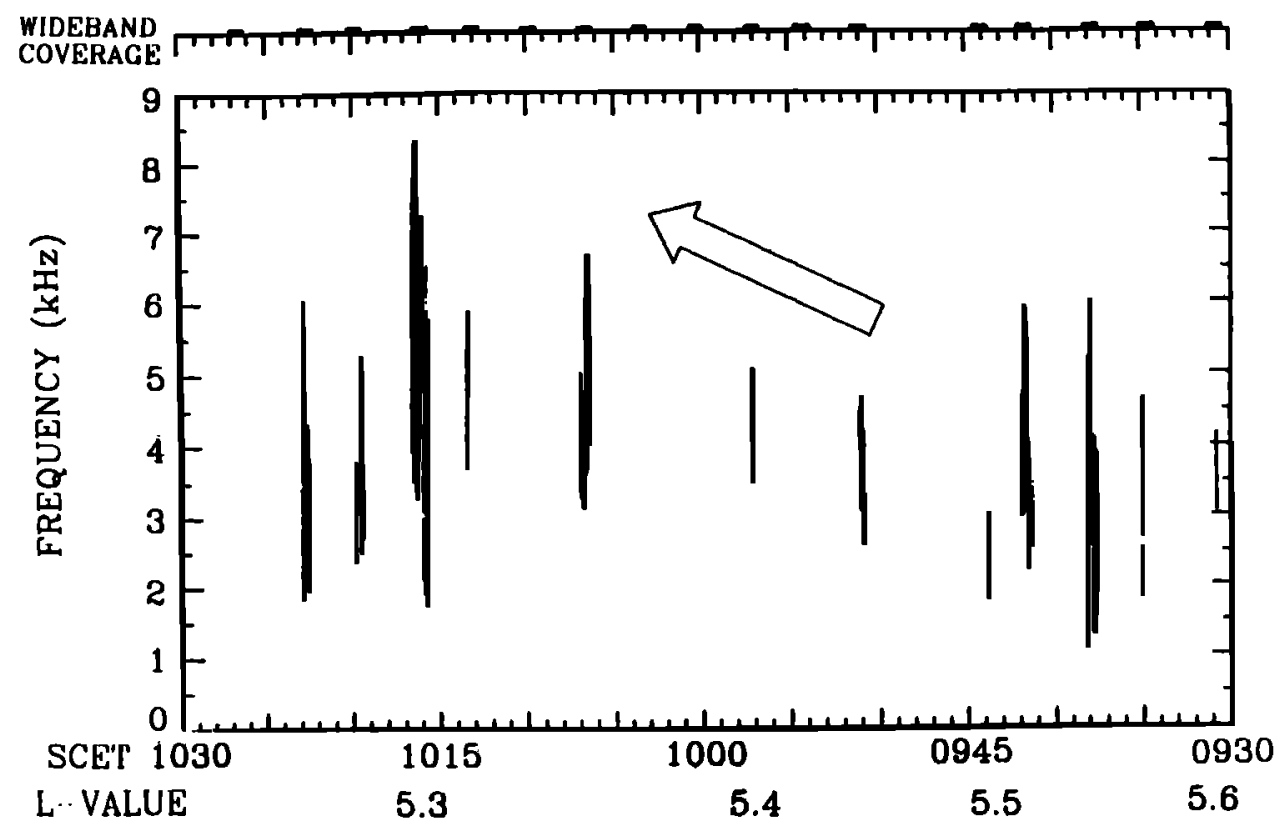

Figure 3. The frequency range of whistlers observed in the cold torus for different $L$ shells and spacecraft event time (SCET). There is a tendency for the upper cutoff frequencies to increase as $L$ decreases over the range $L=5.5$ and $L=5.3$ as indicated by the arrow. Highest frequency $(8 \mathrm{kHz})$ was observed at SCET 1016 . The periods of wideband data coverage are indicated at the top of the Figure [Strayer, 1981]

the inner portion of region $\mathrm{B}$. However, waves launched from higher latitudes at $\Lambda_{g}=44^{\circ}$ (top dotted line) are refracted back to even lower $L$ shells than those for waves launched from $\Lambda_{g}=41.5^{\circ}, 42.5^{\circ}$. This

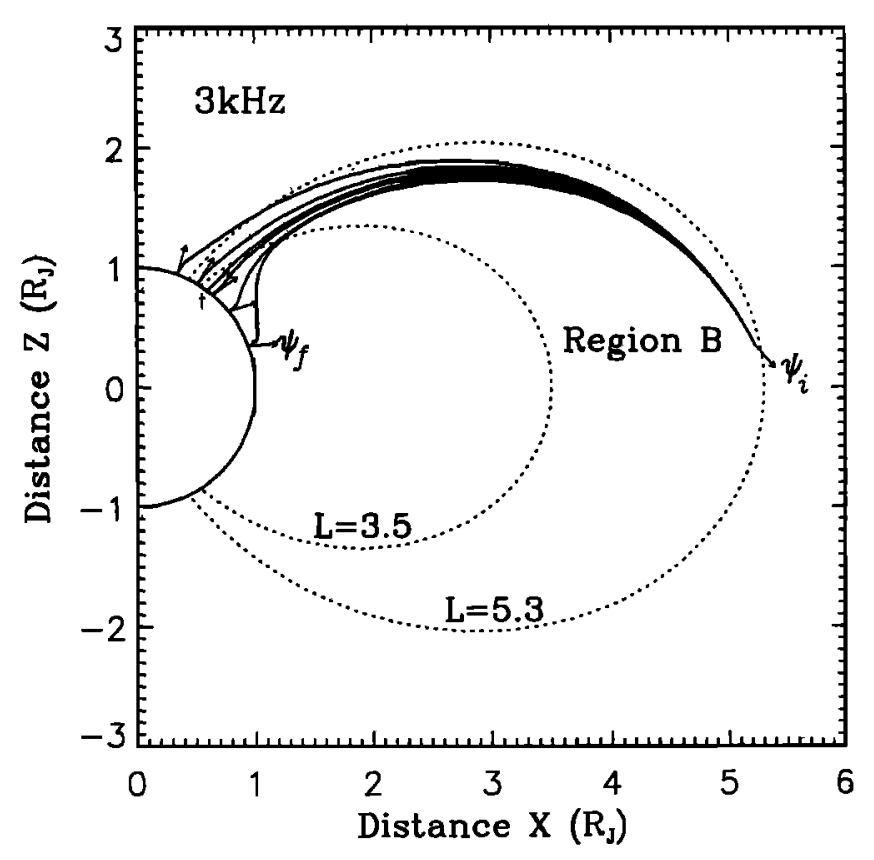

\begin{tabular}{crrrrrr}
\hline$\psi_{i}$ & $141.6^{\circ}$ & $141.7^{\circ}$ & $141.8^{\circ}$ & $141.833^{\circ} \dagger$ & $141.9^{\circ}$ & $142^{\circ}$ \\
\hline$\lambda_{m 0}$ & $20.14^{\circ}$ & $39.13^{\circ}$ & $50.17^{\circ}$ & $53.45^{\circ} \dagger$ & $59.54^{\circ}$ & $70.12^{\circ}$ \\
$\left|\psi_{f}-\psi_{f}^{*}\right|$ & $8.5^{\circ}$ & $4.97^{\circ}$ & $1.2^{\circ}$ & $0.00^{\circ} \dagger$ & $2.71^{\circ}$ & $7.1^{\circ}$ \\
\hline
\end{tabular}

strong refraction of oblique waves results from the low density at middle latitudes in the inner plasmasphere. When waves are launched from much higher latitudes at $\Lambda_{g} \geq 45^{\circ}$ (dashed line), they start to be trapped by the strong lo torus peak at $L=5.7$ and end up in region $\mathrm{A}$. The source location for $8 \mathrm{-kHz}$ region $\mathrm{B}$ whistlers in the OTD model is therefore confined to the narrow range of latitudes $42^{\circ} \leq \Lambda_{g} \leq 43^{\circ}$.

Ray paths for 6-kHz waves (top right panel of Figure 6) do not show the strong refraction toward lower $\mathrm{L}$ shells seen for $8 \mathrm{-kHz}$ waves launched from $\Lambda_{g}=44^{\circ}$. These waves are able to reach region $B$ from a broader range of source locations at lower latitude $\left(40.2^{\circ} \leq\right.$ $\left.\Lambda_{g} \leq 43^{\circ}\right)$. Waves launched from $\Lambda_{g} \geq 44^{\circ}$ propagate into region A. At $4 \mathrm{kHz}$ (bottom left panel), the source location becomes narrower and moves to lower latitude $\left(40^{\circ} \leq \Lambda_{g} \leq 41^{\circ}\right)$. Waves launched between $\Lambda_{g}=41.5^{\circ}$ and $42.5^{\circ}$ are strongly influenced by the steep density gradient between the cold and hot torus and propagate to much higher $L$ shells beyond regions A and B. Waves launched from $\Lambda_{g}=43^{\circ}$ will access region $A$. The propagation characteristics for $2-\mathrm{kHz}$ whistlers are similar

Figure 4. (top) Ray paths of 3-kHz waves with different initial wave angles $\left(\psi_{i}\right)$ launched from the inner portion of region B $(L=5.3)$ back to the ionosphere where the final wave angle is $\psi_{f}$. (bottom) The ionospheric entry latitudes $\lambda_{m 0}$ and the difference between $\psi_{f}$ and $\psi_{f}^{*}$ (the wave angle which is vertical in the ionosphere) are tabulated against $\psi_{i}$. The ray with $\psi_{i}=141.833^{\circ}$, $\lambda_{m 0}=53.45^{\circ}$ is chosen for our study because it corresponds to a vertical wave in the ionosphere. 


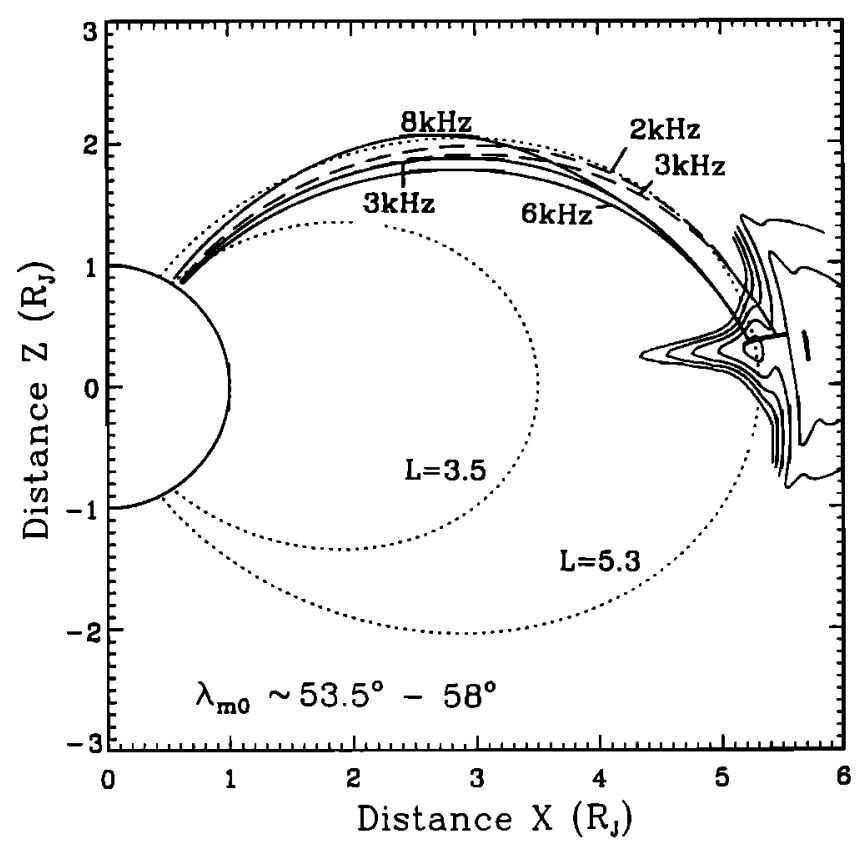

Figure 5. Examples of ray paths of waves observed at SCET $0944(2 \mathrm{kHz}$ to $3 \mathrm{kHz}$ : dashed lines) and SCET $1016(3 \mathrm{kHz}$ to $8 \mathrm{kHz}$ : solid lines) (see Figure $3)$. Eight-kilohertz waves are noticeably refracted at middle latitudes $\left(\lambda_{m}=30^{\circ}-45^{\circ}\right)$ by the density minimum at $L=5.3$ and arrive near the inner portion of region $B$. Lower-frequency waves (dashed lines) tend to be refracted by the Io torus density gradient and travel to the outer portion of region $B$.

to those at $4 \mathrm{kHz}$. The only notable difference occurs because the ray paths of waves with lower frequencies deviate more from the ambient magnetic field lines and are more easily trapped by high density in the warm torus. None of these waves propagate along field lines. They cross a large range of $L$ shells and originate from a source location at much lower $L$ shell in the ionosphere. Most of the cross- $L$ transport occurs at high latitude, but the ray path of low-frequency ( 2 and $4 \mathrm{kHz}$ ) waves exhibits additional refraction by the strong radial density gradient near the equator $\left(\lambda_{m} \leq 10^{\circ}\right)$ which carries such waves into the outer portion of region $B$.

Figure 6 shows how electron densities at middle to high latitudes in the inner plasmasphere control the access of high-frequency whistlers to region $\mathrm{B}$. The ray tracing results constrain the minimum electron density along the ray path to values between 3 and $5 \mathrm{~cm}^{-3}$. The sensitivity of the ray paths to small changes in the plasmaspheric density is explored in Figure 7, which shows ray paths of $8-\mathrm{kHz}$ (observed) and $9-\mathrm{kHz}$ (unobserved) waves launched from $\lambda_{m 0}=58^{\circ}$. The top panel shows that a $20 \%$ increase (dashed line) in this plasmaspheric density $\left(N=1.2 N_{0}\right)$ would cause $8-\mathrm{kHz}$ waves (dashed line) to be carried into the outer portion of region B. Eight-kilohertz waves were not observed in this location, indicating a firm upper limit of the plasmaspheric density at high latitudes. Alternatively, for a $20 \%$ decrease in this density $\left(N=0.8 N_{0}\right)$,
$8-\mathrm{kHz}$ waves (dash-dotted line) would be unable to access any part of region $B$. This places a lower limit on the plasmasphere density at high latitudes. The bottom panel shows that for a $20 \%$ increase in this density $\left(N=1.2 N_{0}\right), 9 \mathrm{kHz}$ waves (dashed line) would gain access the inner portion of region $\mathrm{B}$. When density is further increased $\left(N=1.5 N_{0}\right), 9-\mathrm{kHz}$ waves can even access the outer portion of region $B$. The absence of $9-\mathrm{kHz}$ waves anywhere in region $\mathrm{B}$ places a firm upper limit on the plasma density. Although the ray paths are sensitive to the precise model used to simulate density in the inner plasmasphere, it is safe to conclude that the electron density at high latitude is limited to values near a few cubic centimeters.

\section{Discussion and Conclusions}

On the basis of detailed ray path calculations with a comprehensive electron density model for the inner Jovian magnetosphere, the observed characteristics of region $B$ whistlers can be interpreted briefly as follows. The dispersion of signals which reach region $\mathrm{B}$ near $L=$ 5.3 is always low, $D \approx 44 \mathrm{~s} \mathrm{~Hz}^{1 / 2}$, which is consistent with the observed dispersions near SCET 1016 (Figure 3 ). In contrast to the density peaks in the warm torus at $L=5.7$ and $L=5.9$ which guide waves to region $\mathrm{A}$ and C [Wang et al., this issue], plasma in the cold torus does not extend to sufficiently high latitude to guide waves into region B. However, electron density in the inner plasmasphere at middle and high latitudes around $L=5.3$ does control the access of whistlers to region $\mathrm{B}$. The minimum density along the ray path needs to be high enough to allow waves to access the cold torus. In our earlier work [Wang et al., 1995], the model electron density was insufficient to allow highfrequency whistlers into this region. The broad spatial distribution of whistler events in region B from $L=\mathbf{5 . 3}$ to $L=5.6$ can be explained by the effects of refraction by the density gradients between the cold torus and the warm torus (Figures 1, 5, and 6). Waves with lower frequencies are more easily refracted to access the outer portion of region $B$.

Whistlers propagate significantly across field lines along their path from the source location in the ionosphere $(3 \leq L \leq 4)$ to the equatorial observation regions $(5.3 \leq L \leq 5.6)$ in the cold Io torus (Figure 6). Source latitudes for waves of all frequencies observed in region $\mathrm{B}$ range from $40^{\circ}$ to $45^{\circ}$ Jovigraphic. This range is consistent with the location of optical lightning which was observed on Voyager over the range from $30^{\circ}$ to $55^{\circ}$ Jovigraphic latitudes [Cook et al., 1979; Magalhães and Borucki, 1991]. The fact that most whistlers were observed in region $B$ may correspond to the strong peak in lightning occurrence near $49^{\circ}$ Jovigraphic. Although these lightning events were detected at night (3 hours after the whistler events), natural lightning events should not be sensitive to longitude [Magalhães and Borucki, 1991]. The exact source location in the ionosphere cannot be derived using our simple OTD model. Changes in parameters of the OTD model from 

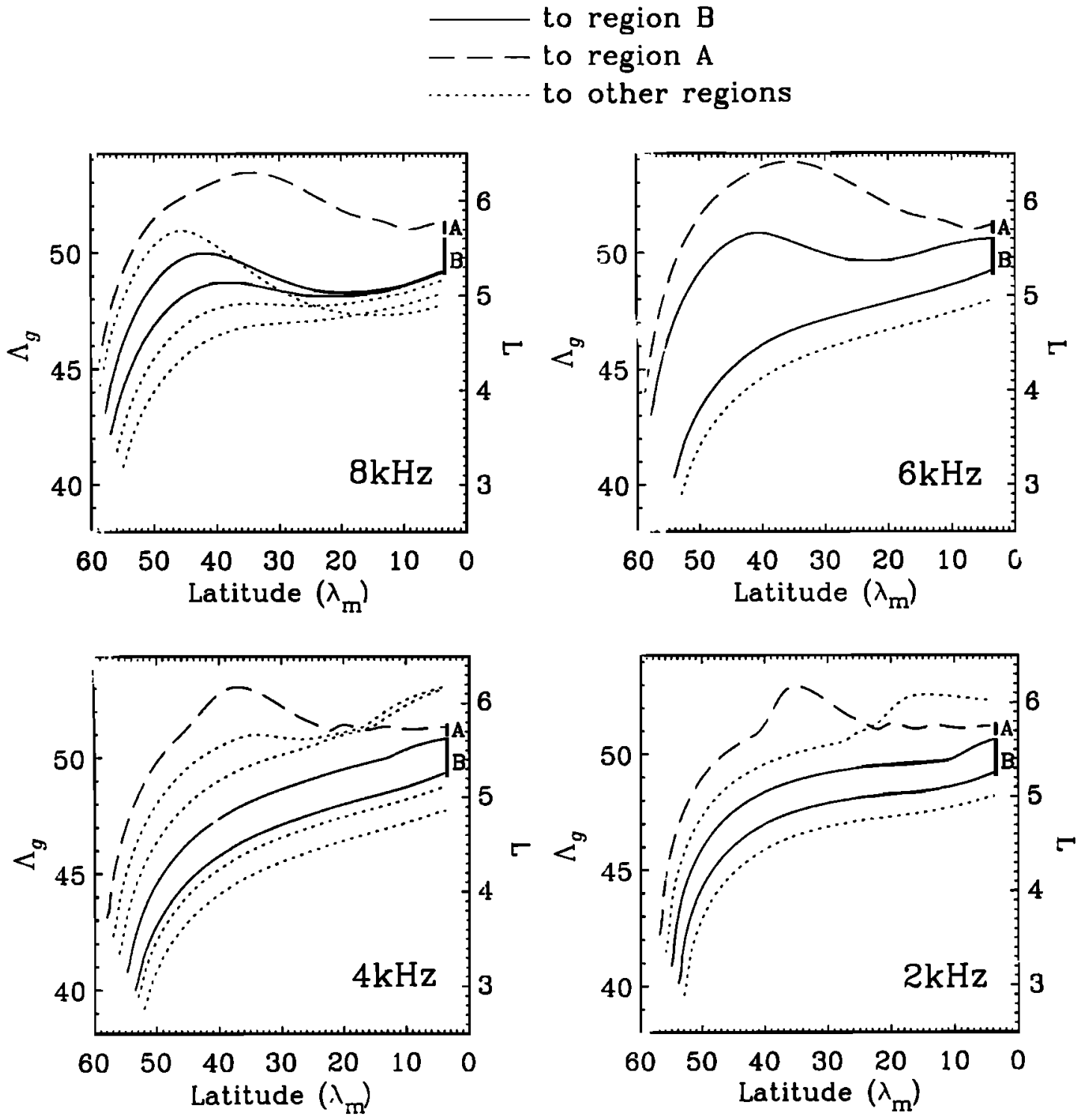

Figure 6. Ray paths plotted as a function of invariant Jovigraphic latitude $\left(\Lambda_{g}\right)$ versus Jovimagnetic latitude $\left(\lambda_{m}\right)$. At $8 \mathrm{kHz}$ (top left), only waves launched from a limited range of latitudes $43^{\circ} \leq \Lambda_{g} \leq 44^{\circ}$ are able to propagate to region $B$. The access to region $B$ at other frequencies is indicated in the remaining panels. The source latitudes of lower frequency waves moves to lower Jovigraphic latitude. The source in the ionosphere occurs at a significantly lower L shell than the observation location.

Acuña et al., 1983] and the inclusion of quadrupole and octupole terms near the planet would change the source location. However, it is worth noting that the source latitudes from the OTD model are well within the range of optical lightning events.

The trend of the observed upper cutoff frequencies exhibited in Figure 3 is a direct consequence of the electron density structure of the inner plasmasphere. Decrease in electron density with increasing $L$ shell provides a plasma environment which allows lowerfrequency waves to access the outer portion of region $\mathrm{B}$, but confines the access of higher-frequency waves to the inner portion. The model for the Jovian plasmasphere is a modification of the density model introduced by Divine and Garrett [1983] which has only two free parameters, limiting our ability to match the upper cutoff frequency; basically, we can only attempt to fit the trend from the inner to the outer part. As Figure 3 shows, the upper cutoff frequencies around $L=5.6$ are higher. This may be a consequence of some mixture of plasma from the cold torus and the warm torus near this location and will not affect our interpretation of the upper cutoff frequency trend at lower $L$ in the cold torus.

Finally, the constraint on electron density (3 - 5 $\mathrm{cm}^{-3}$ ) at middle and high latitudes along $L=5.3$ is quite firm, since it cannot be too high or too low to interpret the confined observations of $8-\mathrm{kHz}$ waves 

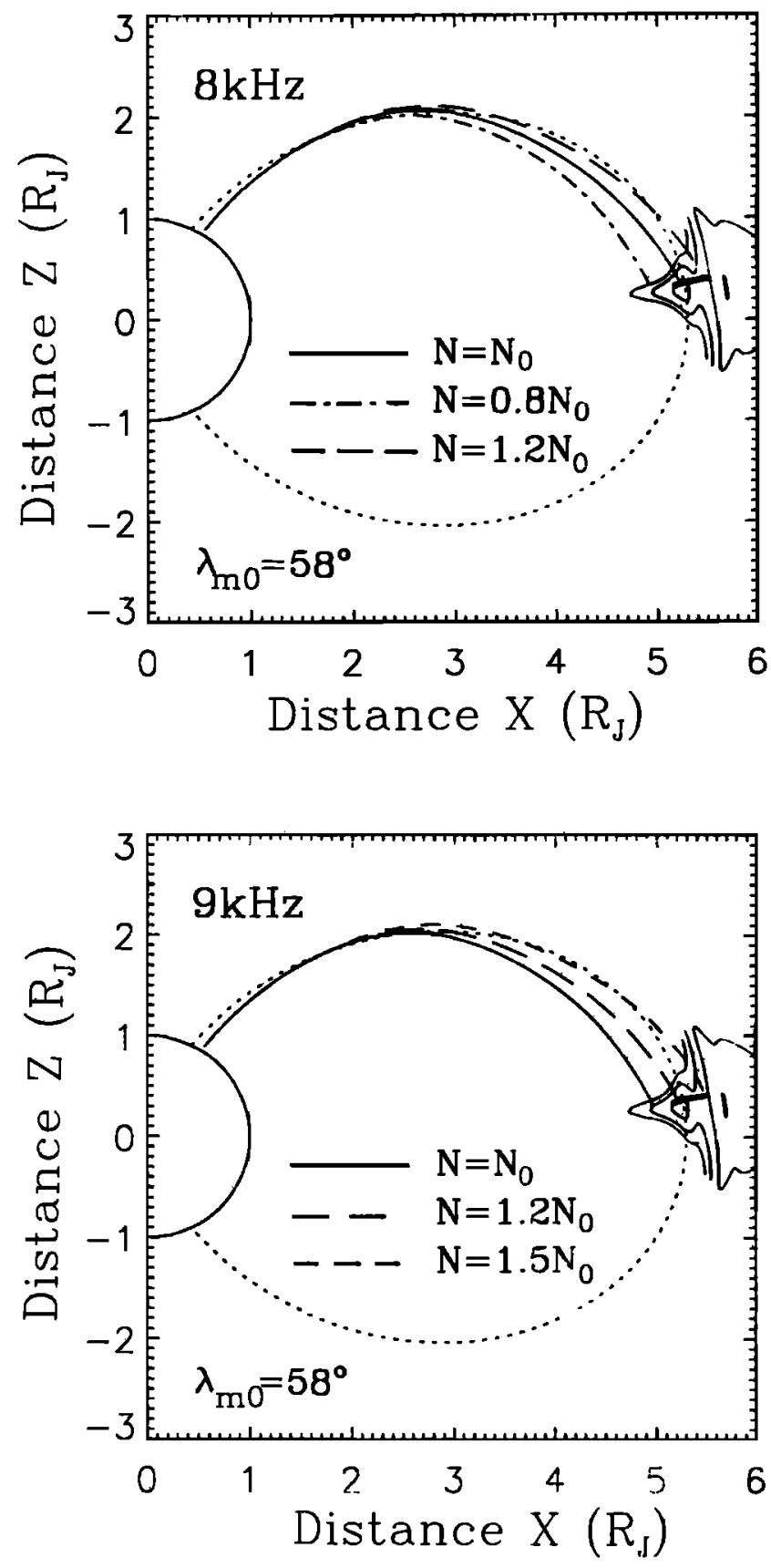

Figure 7. (top) Sensitivity of the access of $8-\mathrm{kHz}$ waves to region $B$ (bold solid line) to variations in the inner plasmasphere electron density $\left(N_{0}\right.$ denotes nominal density). (bottom) Nine-kilohertz waves should be able to gain access to region $\mathrm{B}$ if the inner plasmasphere electron density were increased by $20 \%$ above our nominal model.

around $L=5.3$ in region B (Figure 7). The constraint on electron density for the inner plasmasphere is also consistent with the study on Jovian decametric radio emission [Melrose and Dulk, 1991], which requires less than $5 \mathrm{~cm}^{-3}$ to interpret the observed radiation, implying that the presence of whistlers in region $B$ provides a sensitive bound on the electron density in a region where no spacecraft have ever been.
Acknowledgments. The authors thank J. Groene for assistance with Voyager whistler data and also F. Bagenal for valuable discussion concerning the Jovian inner plasmasphere. Voyager 1 PWS data were provided by the Outer Planets Subnode of the Planetary Plasma Interactions Node of the Planetary Data System. Computational resources were provided by the San Diego Supercomputing Center. This work was supported by NASA grant NAG5 6265 and NSF grant ATM97 29021.

The Editor thanks Michael L. Kaiser and another referee for their assistance in evaluating this paper.

\section{References}

Acuña, M. H., K. W. Behannon, and J. E. P. Connerney, Jupiter's magnetic field and magnetosphere, in Physics of the Jovian Magnetosphere, edited by A. J. Dessler, pp. 1-50, Cambridge Univ. Press, New York, 1983.

Bagenal, F., Empirical model of the Io plasma torus: Voyager 1 measurements, J. Geophys. Res., 99, 11,043, 1994.

Cook, A.F., T. C. Duxbury, and G. E. Hunt, First results on Jovian lightning, Nature, 280, 794, 1979.

Crary, F. J., F. Bagenal, J. A. Ansher, D. A. Gurnett, and W. S. Kurth, Proton density and thermal anisotropy of the Io plasma torus derived from whistler wave dispersion, J. Geophys. Res., 101, 2699, 1996.

Divine, N., and H. B. Garrett, Charged particle distributions in Jupiter's magnetosphere, J. Geophys. Res., 88, 6889, 1983.

Gurnett, D. A., and F. L. Scarf, Plasma waves in the Jovian magnetosphere, in Physics of the Jovian Magnetosphere, edited by A. J. Dessler, pp. 285-316, Cambridge Univ. Press, New York, 1983.

Gurnett, D. A., P. R. Shaw, R. R. Anderson, and W. S. Kurth, Whistlers observed by Voyager 1: Detection of lightning on Jupiter, Geophys. Res. Lett., 6, 511, 1979.

Gurnett, D. A., F. L. Scarf, W. S. Kurth, R. R. Shaw, and R. L. Poynter, Determination of Jupiter's electron density profile from plasma wave observations, J. Geophys. Res., 86, 8199, 1981.

Helliwell, R. A., Whistlers and Related Ionospheric Phenomena, Stanford Univ. Press, Stanford, Calif., 1965.

Horne, R. B., Path-integrated growth of electrostatic waves: The generation of terrestrial myriametric radiation, $J$. Geophys. Res., 94, 8895, 1989.

Kurth, W. S., B. D. Strayer, D. A. Gurnett, and F. L. Scarf, A summary of whistlers observed by Voyager 1 at Jupiter, Icarus, 61, 497, 1985.

Magalhāes, L. A., and W. J. Borucki, Spatial distribution of visible lightning on Jupiter, Nature, 349, 311, 1991.

Mei, Y., R. M. Thorne, and F. Bagenal, Analytical model for the density distribution in the Io plasma torus, $J$. Geophys. Res., 100, 1823, 1995.

Melrose, D. B., and G. A. Dulk, On the elliptical polarization of Jupiter's decametric radio emission, Astron. Astrophys., 249, 250, 1991.

Menietti, J. D., and D. A. Gurnett, Whistler propagation in the Jovian magnetosphere, Geophys. Res. Lett., 7, 49, 1980.

Sentman, D. D., and C. K. Goertz, Whistler mode noise in Jupiter's inner magnetosphere, J. Geophys. Res., 83, $3152,1978$.

Strayer, B. D., Observations of Whistlers from Jupiter by Voyager 1, masters thesis, University of Iowa, Iowa City, 1981.

Strobel, D. F., and S. K. Atreya, Ionosphere, in Physics of the Jovian Magnetosphere, edited by A. J. Dessler, pp. 51-67, Cambridge Univ. Press, New York, 1983.

Wang, K., R. M. Thorne, and R. B. Horne, The propagation 
characteristics and Landau damping of Jovian whistlers in the Io torus, J. Geophys. Res., 100, 21,709, 1995.

Wang, K., R. M. Thorne, R. B. Horne, and W. S. Kurth, Constraints on Jovian plasma properties from a dispersion analysis of unducted whistlers in the warm Io torus, $J$. Geophys. Res, this issue.

R. B. Horne, British Antarctic Survey, Madingley Road, Cambridge, CB3 OET, England. (e-mail: R.Horne@bas.ac.uk)

W. S. Kurth, Department of Physics and Astronomy, Uni- versity of Iowa, Iowa City, IA 52242-1479. (e-mail: williamkurth@uiowa.edu)

R. M. Thorne, Department of Atmospheric Sciences, University of California at Los Angeles, CA 90095-1565. (email: rmt@jupiter.atmos.ucla.edu

K. Wang, M/S 169-506, Jet Propulsion Laboratory, 4800 Oak Grove Drive, Pasadena, CA, 91109-8099. (e-mail: kwang@jplsp.jpl.nasa.gov)

(Received September 22, 1997; revised March 2, 1998; accepted March 16, 1998.) 